\title{
Role of Supine and Standing Radiography in Vertebroplasty for Osteoporotic Compression Fractures"
}

\author{
Jae Hyun Park, Sang Hyun Kim, Noh Sung Hyun, Pyung Goo Cho \\ Department of Neurosurgery, Ajou University Hospital, Ajou University School of Medicine, Suwon, Republic of Korea
}

Corresponding author:

Pyung Goo Cho

Department of Neurosurgery, Ajou

University Hospital, Ajou University

School of Medicine, 164, World

Cup-ro, Yeongtonggu, Suwon

16499, Republic of Korea

Tel: $+82-31-219-5230$

Fax: +82-31-219-5238

Email: nsdrcpg@gmail.com
Received: July 27, 2021

Revised: September 23, 2021

Accepted: September 29, 2021

\begin{abstract}
Objective: To confirm the benefits of vertebroplasty (VP) in selected patients with acute vertebral compression fracture (VCF) and analyze whether the study of a weight dynamic plain lateral radiograph would help in making decisions to perform VP. Using retrospective analysis, we aimed to determine the radiological characteristics of patients benefiting from VP.

Methods: Data were collected from 54 patients (age, 56-97 years) diagnosed with osteoporosis and compression fractures between December 2013 and January 2018. Each patient was hospitalized with $\geq 2$ weeks of absolute bed rest (ABR) and treated for osteoporosis. Plain lateral supine radiography of the fractured spine was performed prior to diagnosis, and both supine and standing spinal radiographs were taken 1 and 2 weeks after ABR. Patients were categorized into the VP and non-VP groups 2 weeks after $A B R$.

Results: Although patients with worse pain and functional progress were selected to undergo VP, patients in the VP group presented better outcomes in the fourth week of evaluation than those in the non-VP group. In a retrospective analysis of the radiographic study, changes in the compression rate between supine and standing (weight-bearing) $\mathrm{X}$-rays $(\triangle \mathrm{CR})$, showed a statistically significant correlation with the patient's outcome in the second week.

Conclusion: VP was found to be an effective management option for patients with VCF. A weightbearing radiographic study of VCFs provided valuable information on patient selection for VP. Therefore, patient selection based on subjective surveys and radiological studies to determine the benefits of VP could be a beneficial management strategy.
\end{abstract}

Key Words: Fractures, compression; Osteoporotic fractures; Radiography; Vertebroplasty; Weight-bearing

\section{INTRODUCTION}

Vertebral compression fracture (NCF) is a common type of fracture of the skeletal system ${ }^{20}$. Because most of its etiology is osteoporotic, approximately 1.4 million cases are recorded annually, and its prevalence is increasing as the population ages worldwide ${ }^{13)}$.

VCFs can result in severe pain, spinal misalignment, kyphosis, increased days of bed rest, decreased pulmonary function, inability to perform activities of daily living, depression, and marked reduction in the quality of life and are associated with morbidity and even mortality in patients with osteoporosis ${ }^{24)}$. Thus, adequate prevention and management are essential for reducing these burdens ${ }^{30}$.

The normal evolution of spinal VCF is reported to be spontaneous consolidation, with progressive decrease in pain during a 6 to 8-week period $^{211}$. The current guidelines for standard treatment suggest conservative medical care with rest, analgesia, and external bracing ${ }^{22)}$. In addition, with advancements in medical options, including pharmaceuticals, such as teriparatide, romosozumab, and denosumab, conservative osteoporosis treatment has become more effective ${ }^{17}$. However, conservative care can also exacerbate bone deminerali- zation, and patients may have to tolerate the adverse effects of anti-inflammatory drugs and analgesics ${ }^{28)}$. Furthermore, patients can become dissatisfied with prolonged bed rest because it may cause pneumonia, urinary infection, bedsores, and deep venous thrombosis ${ }^{26)}$. Vertebroplasty (NP) or open surgery, which is performed to prevent abnormal motion, restores vertebral height and corrects kyphosis and may be considered in patients refractory to medical therapy ${ }^{1,9)}$.

VP, in particular, was first developed in France by Galibert et al. ${ }^{11)}$ in 1987. It is an internal fixation method that utilizes medical cement injections, thereby uniting the fragments and preventing fracture fragment motion ${ }^{1,11)}$. This fixation principle in acute fracture pain management has also been well accepted in the management of other skeletal fractures ${ }^{18}$. However, with the publication of the INNEST study by Kallmes et al. ${ }^{15}$, the Australian trial by Buchbinder et al. $^{4}$, and the VERTOS IV trial ${ }^{10}$, the merits of VP are under debate. Nevertheless, as a relatively non-invasive, low-risk procedure, which is believed to provide immediate and durable pain relief and improve function in patients with painful VCFs, VP is the main method in the management of osteoporotic and malignant vertebral fractures ${ }^{5,6,8)}$.

Selecting a patient who is refractory to conservative care is key 
to maximizing the potential benefit of $\mathrm{VP}^{3)}$. Although the current clinical guideline recommendations have generally been inconsistent and based on clinical and subjective parameters, there is a limit to determining the viability of VP based on clinical status alone ${ }^{22,25)}$. Therefore, our study aimed to determine whether radiological studies provide information on the structural instability of the lesion and contribute to patient selection for VP.

\section{MATERIALS AND METHODS}

This retrospective cohort study was conducted in accordance with the Declaration of Helsinki, and the protocol was approved by the ethics committee prior to study initiation (IRB No. DMC 201902-001).

\section{Data Collection}

The study was based on data collected from December 2013 to January 2018, comprising 129 patients hospitalized in the emergency room (ER) with acute back pain for $<1$ week. Among the 129 patients, 54 were finally induded in the analysis based on the indusion and exclusion criteria, and their data were collected from medical charts, radiological images, and surgeons' records. The inclusion criteria were as follows: (1) acute VCF on magnetic resonance imaging (MRI); (2) age, 50 to 100 years; and (3) osteoporosis diagnosis with bone mineral density (BMD) (T-score $\leq-2.5)$ on a dual energy $X$-ray absorptiometry. The exclusion criteria were as follows: (1) potential harm to patients with cardiopulmonary conditions (pneumonia, chronic obstructive pulmonary disease, and chronic heart failure) in the absolute bed rest (ABR) state; (2) threecolumn vertebral fracture; and (3) pathological lumbar spine conditions (tumor or infection).

\section{Patient Management Protocol and Assessment Tool}

All patients were hospitalized from the ER immediately after an acute VCF diagnosis with newly developed back pain. Hospital care was required for all patients for at least 4 weeks, and 2 weeks of $A B R$ was mandated, even during mealtimes or while using the toilet. Opioid and/or non-steroidal anti-inflammatory drugs were administered to control pain. All patients were injected with teriparatide (20 $\mu \mathrm{g}$ daily, SQ) and treated with calcium and vitamin D supplements for osteoporosis.

Patients eligible for VP were selected after 2 weeks of conservative care with mandated ABR. Specifically, patients who were refractory to conservative care, primarily defined as those with a visual analog scale (NAS) score $\geq 5$ or Oswestry Disability Index (ODI) score $\geq 30$ were selected for VP. Among patients with a VAS score of 5, two patients showed prominent improvement in the ODI score, reporting a $>5$-point decrease in the recent week. As they have shown progress, they were deemed responsive and continued to receive conservative care. Regardless of the selection, conservative care under hospitalization continued for another 2 weeks or more without mandatory $A B R$, totaling 4 weeks of hospital care at least.

All VP procedures were performed percutaneously by a single neurosurgeon using the +transpedicular method. Patients were then categorized into VP and non-VP groups for the retrospective analysis of radiological and clinical status to determine the characteristics of patients refractory to conservative care and the predictive values that the patients eligible for VP would show. In particular, fracture level, age, sex, body mass index, and bone mineral density (BMD) of the two groups were analyzed.

\section{Radiological Assessment}

On the day of admission, MRI and BMD evaluations were performed to confirm the diagnosis of VCF. Initially, supine radiographs of the lateral spine view were obtained from each patient. Radiographs of the lateral view of the non-weight-bearing supine and weightbearing standing positions under rigid thoracolumbosacral orthosis brace support were taken during the first and second week after hospitalization with ABR. Four weeks after the date of hospitalization, only radiographs of the lateral view of the non-weight-bearing supine position were studied (Fig. 1).

Vertebral body stability was evaluated by comparing the compression rate (CR) with the change in vertebral height of the fractured bone and lordotic angle (LA) in the supine and standing lateral radiographs 1 and 2 weeks after admission. Thereafter, CR was used to calculate the estimated percent ratio of compressed lost vertebral body height to the estimated normal anterior height. The calculation formula is as follows (Equation 1; Fig. 2):

$$
\begin{gathered}
\text { Equation 1. Compression rate (\%) formula } \\
C R(\%)=\left(1-\frac{2 * \text { anterior height of fractured body }}{(\text { anterior heigh of upper body }+ \text { anterior height of lower body })}\right) \times 100
\end{gathered}
$$

LA was measured using the Cobb's angle made by the upper and lower endplates of the fracture body (Fig. 2). $\triangle \mathrm{CR}$ was defined as the change in the compression value between the supine (nonweight-bearing) and standing (weight-bearing) radiographs. Specifically, $\Delta \mathrm{CR}$ was calculated by subtracting the $\mathrm{CR}$ value in the standing

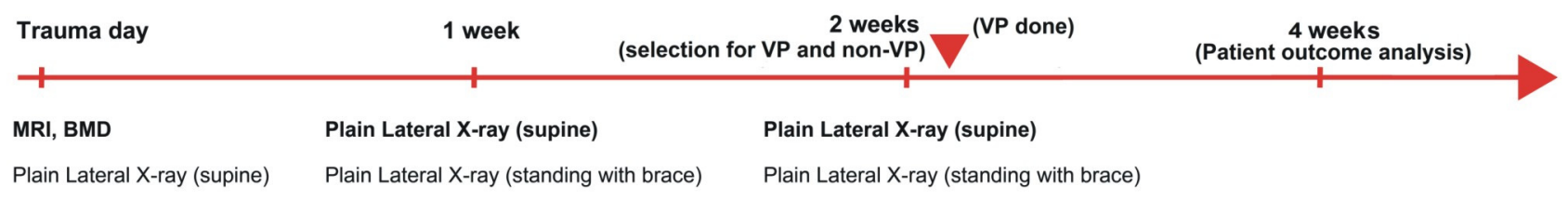

Fig. 1. Imaging timeline of the study. MRI: magnetic resonance imaging; BMD: bone mineral density; VP: vertebroplasty. 
position from the CR value in the supine position (Equation 2).

$$
\begin{gathered}
\text { Equation 2. } \Delta \mathrm{CR} \text { formula } \\
\Delta \mathrm{CR}=(C R \text { supine })-(C R \text { standing })
\end{gathered}
$$

The loss of vertebral height and bone edema on MRI was confirmed by radiology specialists to determine compression fractures. Measurements of the radiological features were then performed by two neurosurgeons, and the average value was used for analysis.

\section{Clinical Assessment}

We used the VAS and ODI scores at the initial assessment and 1,2 , and 4 weeks after admission. VAS scores for back and leg pain were measured separately on a scale of 1 to 10 , with 0 representing no pain and 10 representing the most severe pain imaginable. ODI scores are one of the principal condition-specific outcome measures used in spinal disorder management using a 50-point questionnaire, with 0 representing no disability and 50 representing bed-boundedness or exaggeration of symptoms. Clinical scores of VAS and $\mathrm{ODI}$ were surveyed by one nurse specializing in neurosurgery; both score surveys were initially investigated 1 and 2 weeks after ABR and 4 weeks after hospitalization. Patients who underwent VP were additionally surveyed for comparison a day after the procedure.

\section{Statistical Analysis}

All analyses were performed using the IBM SPSS 22 program (SPSS Inc., Chicago, IL, USA), and differences were considered statistically significant at $p$-value less than 0.05 . We used the MannWhitney nonparametric statistical test corresponding to the $\chi^{2}$ test to compare fracture levels, follow-up periods, VAS scores, ODI

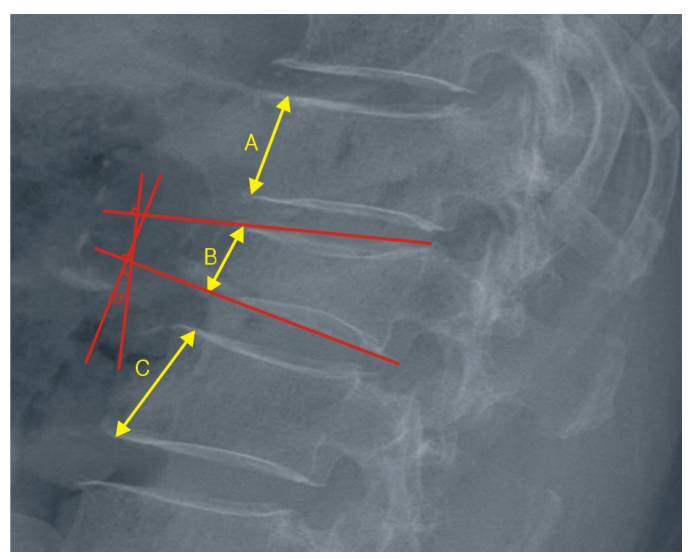

Fig. 2. Compression rate (\%) calculation. Compression rate is calculated by subtracting the anterior body height of the fractured body (B) from the average anterior height of the upper (A) and lower (C) vertebral bodies and then dividing the difference by the average anterior height of the upper and lower vertebral bodies, which is converted into percentage. Lordotic angle $\left(a^{\circ}\right)$ was measured by the angle made by the upper and lower endplates of the fracture body. scores, $\mathrm{CR}$ and $\triangle \mathrm{CR}$, patient characteristics, and baseline features between the VP and non-VP groups. Simultaneously, the Wilcoxon signed-rank test medians and interquartile ranges were used to evaluate the effect of VP on the VAS score. Statistical correlations between CR, LA, and VAS and ODI scores were then evaluated post hoc using the Spearman rank correlation analysis for nonparametric data analysis.

\section{RESULTS}

\section{Patient Characteristics and Baseline Features}

Overall, 54 patients (46 men and 8 women) were included in the study. Their ages ranged from 56 to 97 years, with a mean age ( \pm standard deviation) of $77.13( \pm 9.28)$ years. VCFs of traumatic origin was present in six cases, whereas VCFs of spontaneous origin were present in the remaining 48 cases. The average T-score measured for $\mathrm{BMD}$ in the spine among patients was $-3.61 \pm 0.75$.

Among the 54 included patients, 30 were selected to undergo VP (NP group), and the remaining 24 continued to undergo conservative care (non-VP group). Fig. 3 shows no significant differences in the fracture level of VCF between the two groups $(p=0.430)$. Moreover, there were no significant differences in the statistical analysis of the two groups in terms of fracture level, sex, BMD, or follow-up period; however, patients in the VP group tended to be significantly older than those in the non-VP group ( $p=0.040$; Table 1).

\section{Radiological Outcomes During Each Follow-up}

The CR and LA of the patients on each period of hospitalization did not show statistically significant changes with time (Table 2, 3).

On initial evaluation, the CR and LA of the VP group were measured to be $23.52 \% \pm 16.49 \%$ and $10.81^{\circ} \pm 6.34^{\circ}$, respectively. In the non-VP group, the CR was $25.36 \% \pm 16.88 \%$ and LA was $11.18^{\circ} \pm$

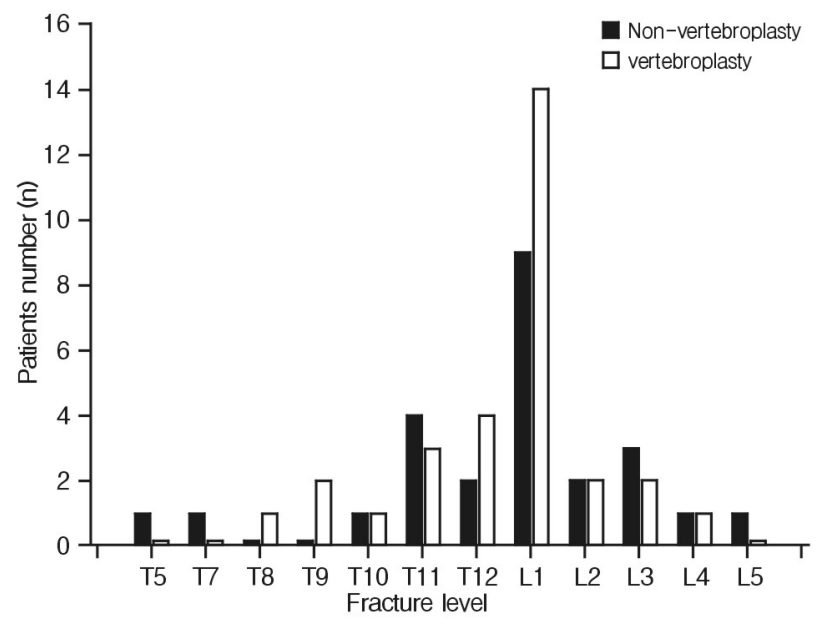

Fig. 3. Fracture levels in the vertebroplasty (VP) and non-VP groups $(p=0.430)$. 
5.12 . No significant differences were found in the CR and LA between the two groups.

At the follow-up after 1 week of $A B R$, the CR in the supine and standing positions was $24.56 \% \pm 13.06 \%$ and $32.58 \% \pm 12.79 \%$, respectively, in the VP group. The corresponding CR measurements in the non-VP group were $27.15 \% \pm 15.66 \%$ and $29.62 \% \pm 14.77 \%$, respectively. The $L A$ in the supine and standing positions were $11.13^{\circ}$ $\pm 5.23^{\circ}$ and $13.78^{\circ} \pm 5.74^{\circ}$, respectively, in the $\mathrm{VP}$ group and $11.40^{\circ}$ $\pm 6.23^{\circ}$ and $12.29^{\circ} \pm 6.14^{\circ}$, respectively, in the non-VP group. Similar to the initial results, no significant differences were observed in the CR and LA between the two groups during the 1-week follow-up period.

At the follow-up after 2 weeks of $A B R$, the $C R$ in the supine and standing positions was $26.40 \% \pm 14.55 \%$ and $37.22 \% \pm 14.33 \%$, respectively, in the VP group and $30.61 \% \pm 17.32 \%$ and $33.65 \% \pm$ $17.17 \%$, respectively, in the non-VP group. The LA in the supine and standing positions was $11.87^{\circ} \pm 5.76^{\circ}$ and $13.08^{\circ} \pm 6.90^{\circ}$, respectively, in the VP group and $12.59^{\circ} \pm 11.87^{\circ}$ and $13.39^{\circ} \pm 6.39^{\circ}$, respectively, in the non-VP group. Similar to the previous results, no significant differences were observed between the two groups regarding CR and LA during the 2-week follow-up period

At the follow-up after 4 weeks of $A B R$, the $\triangle C R$ values of the non-VP and $\mathrm{VP}$ groups were $2.0 \% \pm 6.12 \%$ and $8.2 \% \pm 5.86 \%$, respectively, showing statistical significance $(p=0.010$; Fig. 4, 5). At the 2-week follow-up, the $\triangle \mathrm{CR}$ values of the non-VP and VP groups were $3.04 \% \pm 4.58 \%$ and $9.98 \% \pm 5.61 \%$, respectively $(p=0.010)$.

The CR at each period showed a statistical correlation with the corresponding $\mathrm{LA}$ at the respective follow-up points $(p<0.001)$.

Table 1. Patient characteristics in the VP and non-VP groups

\begin{tabular}{lccc}
\hline \hline Variables & \multicolumn{1}{c}{ Non-VP } & VP & p-value \\
\hline Non-VPNP & 24 & 30 & \\
Sex (Male:Female) & $5: 19$ & $3: 27$ & 0.443 \\
Age (year) & $74.38 \pm 10.40$ & $79.33 \pm 7.77$ & 0.040 \\
BMD (T-score) & $-3.58 \pm 0.75$ & $-3.64 \pm 0.77$ & 0.644 \\
Period of follow-up (months) & $6.33 \pm 3.88$ & $8.88 \pm 4.11$ & 0.132 \\
\hline
\end{tabular}

The data is presented as mean \pm standard deviation or number.

VP: vertebroplasty; BMD: bone mineral density.

Table 2. CR and change in CR on weight bearing in the VP and non-VP groups

\begin{tabular}{lccc}
\hline \hline & Non-VP & VP & p-value \\
\hline Initial CR (\%) & $25.36 \pm 16.88$ & $23.52 \pm 16.49$ & 0.728 \\
1-week supine CR (\%) & $27.15 \pm 15.66$ & $24.56 \pm 13.06$ & 0.614 \\
1-week standing CR (\%) & $29.62 \pm 14.77$ & $32.58 \pm 12.79$ & 0.465 \\
1-week $\Delta$ CR (\%) & $2.47 \pm 6.12$ & $8.02 \pm 5.86$ & 0.010 \\
2-week supine CR (\%) & $30.61 \pm 17.32$ & $26.40 \pm 14.55$ & 0.499 \\
2-week standing CR (\%) & $33.65 \pm 17.17$ & $37.22 \pm 14.33$ & 0.459 \\
2-week $\Delta$ CR (\%) & $3.04 \pm 4.58$ & $9.98 \pm 5.61$ & 0.001 \\
\hline
\end{tabular}

The data is presented as mean \pm standard deviation.

VP: vertebroplasty; CR: compression rate.

Table 3. LA in the VP and non-VP groups

\begin{tabular}{lccc}
\hline \hline & Non-VP & VP & p-value \\
\hline Initial LA $\left(^{\circ}\right)$ & $11.18 \pm 5.12$ & $10.81 \pm 6.34$ & 0.802 \\
1-week supine LA $\left({ }^{\circ}\right)$ & $11.40 \pm 6.23$ & $11.13 \pm 5.23$ & 0.978 \\
1-week standing LA $\left(^{\circ}\right)$ & $12.29 \pm 6.14$ & $13.78 \pm 5.74$ & 0.477 \\
2-week supine LA $\left({ }^{\circ}\right)$ & $12.59 \pm 11.87$ & $11.87 \pm 5.76$ & 0.607 \\
2-week standing LA $\left({ }^{\circ}\right)$ & $13.39 \pm 6.39$ & $13.08 \pm 6.90$ & 0.628 \\
\hline
\end{tabular}

The data is presented as mean \pm standard deviation.

VP: vertebroplasty; LA: lordotic angle. 


\section{Clinical Outcomes}

There were no statistically significant differences between the VP and non-VP groups at the initial evaluation and the 1-week follow-up. On initial evaluation, the VAS and ODI scores in the VP group were $7.63 \pm 0.85$ and $34.03 \pm 0.85$, respectively, whereas those in the non-VP group were $7.66 \pm 0.81$ and $33.91 \pm 2.20$, respectively.

At the 1-week follow-up, the VAS and ODI scores in the VP group were $5.06 \pm 0.63$ and $33.46 \pm 1.99$, respectively, and those in the non-VP group were $5.04 \pm 0.69$ and $33.16 \pm 2.21$, respectively.

At the 2-week follow-up, the VAS and ODI scores in the VP group were $4.96 \pm 0.66$ and $31.23 \pm 1.79$, respectively, and those in the non-VP group were $3.95 \pm 0.55$ and $26.12 \pm 2.07$ ( $p=0.000$ and $\mathrm{p}=0.000)$, respectively.

A day after VP, the VP group showed a statistically significant improvement in the mean VAS score from $4.96 \pm 0.66$ to $2.2 \pm 0.76$ $(p=0.000)$.

At the 4-week follow-up, the VAS and ODI scores in the VP group were $1.8 \pm 0.61$ and $7.43 \pm 2.14$, respectively, whereas those in the non-VP group were $2.83 \pm 1.09$ and $16.70 \pm 1.54(p=0.000$ and $p=0.000$ ), respectively. Both the groups showed gradual improvement, with the VP group showing a significant turnover and faster recovery (Table 4, Fig. 6).

\section{Correlations between Radiological and Clinical Outcomes}

Initial and 1-week follow-up evaluations of the VAS and ODI scores showed no correlation with radiological features.

However, there was a statistically significant correlation between the VAS and ODI scores surveyed in the second week and the $\triangle \mathrm{CR}$ values at the 1 and 2 weeks. Specifically, the $\Delta \mathrm{CR}$ value at
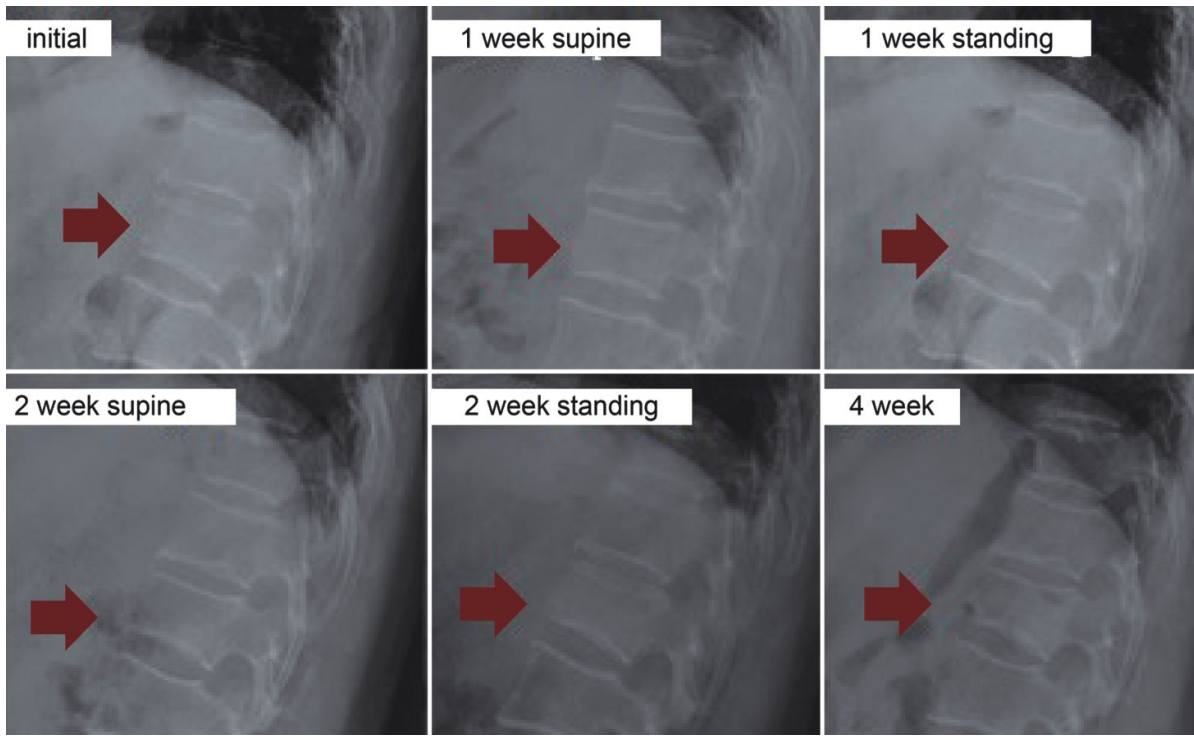

Fig. 4. Radiographic study of a patient in the non-vertebroplasty group. Initial compression rate $=12 \%$, first-week supine compression rate $=14 \%$, first-week standing compression rate $=25 \%$, first-week $\Delta$ compression rate $=11 \%$, second-week supine compression rate $=21 \%$, second-week standing compression rate $=21 \%$, second-week $\Delta$ compression rate $=0 \%$, and fourth-week compression rate $=27 \%$.

Table 4. Pain and functional outcomes based on the VAS and ODI scores between the VP and non-VP groups

\begin{tabular}{|c|c|c|c|c|c|c|}
\hline & \multicolumn{2}{|c|}{ VAS } & \multirow{2}{*}{$\mathrm{p}$-value } & \multicolumn{2}{|c|}{ ODI } & \\
\hline & Non-VP & $\mathrm{VP}$ & & Non-VP & $\mathrm{VP}$ & \\
\hline Initial & $7.64 \pm 0.82$ & $7.63 \pm 0.85$ & 0.904 & $33.91 \pm 2.20$ & $34.03 \pm 0.85$ & 0.792 \\
\hline 1 week & $5.04 \pm 0.69$ & $5.06 \pm 0.63^{*}$ & 0.899 & $33.16 \pm 2.21$ & $33.46 \pm 1.99^{*}$ & 0.598 \\
\hline 2 weeks & $3.95 \pm 0.55^{*}$ & $4.96 \pm 0.66^{*}$ & 0.000 & $26.12 \pm 2.07^{*}$ & $31.23 \pm 1.79^{*}$ & 0.000 \\
\hline 4 weeks & $2.83 \pm 1.09^{*}$ & $1.8 \pm 0.61^{*}$ & 0.000 & $16.70 \pm 1.54^{*}$ & $7.43 \pm 2.14^{*}$ & 0.000 \\
\hline
\end{tabular}

The data is presented as mean \pm standard deviation.

VAS: visual analogue scale; ODI: Oswestry Disability Index; VP: vertebroplasty.

*Significant difference compared to the initial VAS and ODI scores at each follow-up period. 

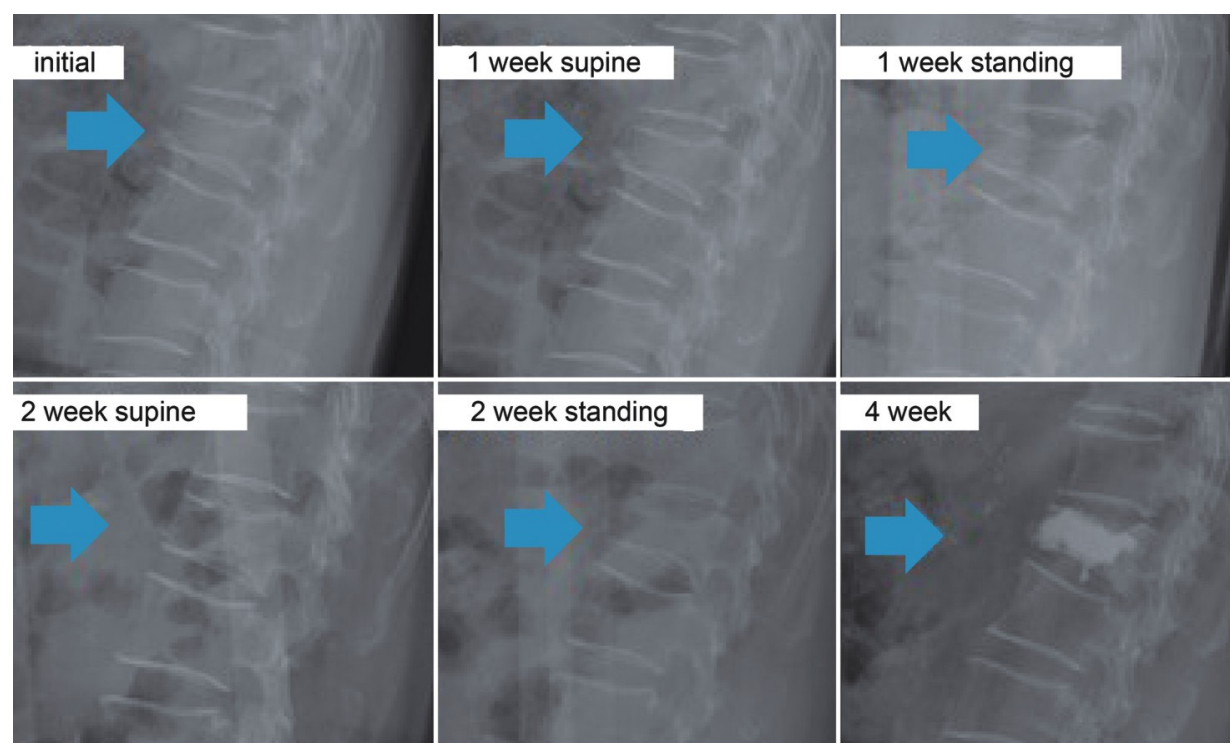

Fig. 5. Radiographic study of a patient in the vertebroplasty group patient. Initial compression rate= $38 \%$, first-week supine compression rate $=38 \%$, first-week standing compression rate $=47 \%$, first-week $\Delta$ compression rate $=9 \%$, second-week supine compression rate $=34 \%$, second-week standing compression rate $=56 \%$, second-week $\Delta$ compression rate $=22 \%$, and fourth-week compression rate $34 \%$.

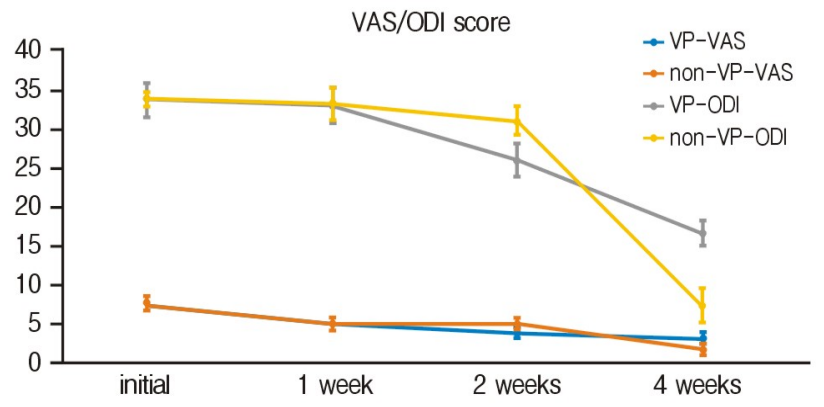

Fig. 6. Pain and functional outcomes in the vertebroplasty (VP) and non-VP groups. The VP group showed better outcomes after undergoing VP. VAS: visual analog scale; ODI: Oswestry Disability Index.

1 week of ABR was correlated with the VAS ( $p=0.008 ; R=0.168$ ) and $\mathrm{ODI}(\mathrm{p}=0.008 ; \mathrm{R}=0.359)$ scores during the second week. Similarly, the $\triangle C R$ value at 2 weeks of $A B R$ showed a correlation with the VAS ( $p=0.001 ; R=0.542)$ and ODI $(p=0.000 ; R=0.572)$ scores at the second week.

Moreover, the $\triangle \mathrm{CR}$ value at 1 week of $\mathrm{ABR}$ showed a correlation with the ODI score at the fourth week with the reversed correlation coefficient $(p=0.001 ; R=-0.431)$, and the $\Delta C R$ value at the second week showed a correlation with the VAS $(p=0.009 ; R=-0.437)$ and $\mathrm{ODI}(\mathrm{p}=0.029 ; \mathrm{R}=-0.370)$ at the fourth week.

\section{DISCUSSION}

As the population ages worldwide, the prevalence of VCFs con- tinues to increase ${ }^{22}$. However, with the rapid pain alleviation provided by chemical/thermal neurolytic effects and fractured vertebrae stabilization, VP has become readily available under local anesthesia, has been deemed relatively safe, and is widely considered a costeffective option ${ }^{2,7,8)}$. Thus, with the increase in the prevalence and benefits of VCF, more patients have been receiving VP for VCF treat ment.

With recent randomized trials showing conflicting results and with the advancing efficacy of conservative care, the benefits of the procedure have been reviewed extensively and repeatedly $4,7,15-17,27$. Although few randomized trials have failed to demonstrate the benefits of VP, several studies have reported that VP is a valid management option, as long as proper patient selection is assured ${ }^{10,12)}$. Consequently, the current recommendations for VP treatment emphasize sufficiently robust patient selection.

However, the criteria recommended by these guidelines are based on clinical and subjective parameters ${ }^{25}$. Korea's national insurance recommends consideration for VP when conservative treatments fail to achieve sufficient pain relief after 2 weeks. Even in-depth reviews from researchers have suggested the criteria for accurate diagnosis, including a shorter pain duration, severe pain, and ununited fracture on $\mathrm{MR}^{19,29)}$. Among these, pain, as the main factor for making decisions to perform VP, depends on the patient's subjective com- plaint. As such, quantifiable parameters have a niche over subjective surveys in making decisions to perform invasive procedures.

Therefore, our analysis hypothesized that the characteristics of patients who may benefit from VP reflect not only pain and functional indices but also radiological features of the affected area.

After 2 weeks of conservative care, we treated eligible patients 
with acute VCF with VP based on pain and functional indices. We then compared the outcome of the VP group to that of the non-VP group (conservative care only), and post hoc analysis was performed on radiographic data collected from the beginning and throughout the period of hospitalization. Although patients with worse pain and functional progress were selected for VP, the symptoms of patients in the VP group showed rapid relief and presented even better outcomes during the 4-week follow-up than those in the non-VP group.

Consistent with the analysis results of a previous study ${ }^{14)}$, our results demonstrated that the calculated CR showed a relationship with the corresponding measured LA. Although a study has shown a relationship between back pain and hyperkyphosis severity ${ }^{23)}$, LA and CR did not show a statistical correlation with the pain and disability indices. According to our results, kyphosis as a radiological feature did not represent a patient's acute symptom. Furthermore, in VCFs, aside from kyphosis, the motion of fractured particles in the vertebra may indicate the patient's status. The $\Delta \mathrm{CR}$, which is the measure of vertebral compression on weight-bearing, at 1 and 2 weeks after ABR showed statistically significant correlations with the VAS and ODI scores in the second week. This indicated that the fractured vertebra had not been healed to withstand the body weight applied, and macroscopic motion of these fractured segments was observed using dynamic radiography ${ }^{1,29}$. Furthermore, the VP group being refractory to conservative care could be attributed to these macroscopic and microscopic motions in the fractured vertebra, resulting in larger $\Delta C R$ values ${ }^{18}$. Therefore, cement augmentation in these patients should be beneficial.

In the VP group, the analysis of $\triangle \mathrm{CR}$ values at 1 and 2 weeks after ABR showed a correlation with patient outcomes at the fourth week using a reversed correlation coefficient. This relationship was considered to be due to the selection of favorable outcomes of VP.

Comparing the radiological features of the two groups, $\triangle \mathrm{CR}$ of the VP group at the 1- $(8.02 \pm 5.86 \%)$ and 2-week periods (9.98 \pm $5.61 \%)$ showed a statistically significant increase. The VAS score also showed a correlation with $\triangle \mathrm{CR}$ at the corresponding periods; however, the differences between the two groups were close to 1 point (VP group, 4.967 \pm 0.66 ; non-VP group, 3.95 \pm 0.55 ). Relying on one point of a subjective survey for making a decision in invasive surgery is incomplete in many aspects; thus, considering quantitative values can strengthen the decision-making process.

Moreover, though patients were categorized into VP and nonVP groups based on the VAS and ODI scores of the $2^{\text {nd }}$ week, no statistical differences of the VAS and ODI scores were shown in data during the initial and even the $1^{\text {st }}$ week of conservative care. In contrast, by retrospective analysis, the $\Delta C R$ value of the future VP group showed differences in the $1^{\text {st }}$ week. Since patient selection was made by $2^{\text {nd }}$ week's VAS and ODI scores, and that these parameters surveyed on $1^{\text {st }}$ week did not predict the $2^{\text {nd }}$ week's selection, $1^{\text {st }}$ week's $\Delta \mathrm{CR}$ showing statistical differences gives $\Delta \mathrm{CR}$ the potential to be useful as a predictive value in future patient management and selection for VP.

Though we found a statistical difference in the $\Delta C R$ value with patients who showed worse clinical outcomes after 2 weeks of con- servative care, and these patients gained benefits from VP, our study is limited by its retrospective design. We believe a prospective cohort study with the $\Delta \mathrm{CR}$ value as a patient selection tool could elucidate the matter. In addition, the study had a relatively small sample size and did not involve a sham procedure to eliminate a placebo. Moreover, although hospitalization care and evaluation for patient outcome were continued for at least 4 weeks, measurement and analysis of $\mathrm{CR}$ and $\triangle \mathrm{CR}$ were only performed for the 1-week and 2-week radiograph images. Although the results were considered to be relatively reliable, as all patients received care under the same and controlled environment with $A B R$ as a principal to eliminate bias, a study with a larger cohort is needed to confirm these results. Furthermore, the varying bone quality of the patients was quantified using BMD only. Further evaluation (such as performing quantitative computed tomography) would help reduce this bias.

\section{CONCLUSION}

The decision to perform VP in patients with VCF is challenging. With conflicting results and indistinctive evidence, patient selection is considered key to VCF management. Although the current clinical guidelines are generally inconsistent, both clinical parameters and radiological features are helpful tools for determining the need for invasive intervention. Thus, radiological features provided by weightbearing radiographs could benefit patient selection and ensure more favorable outcomes, especially for those with difficulties in responding to the survey.

\section{CONFLICTS OF INTEREST}

No potential conflict of interest relevant to this article was reported.

\section{REFERENCES}

1. Barr JD, Barr MS, Lemley TJ, McCann RM: Percutaneous vertebroplasty for pain relief and spinal stabilization. Spine (Phila Pa 1976) 25:923-928, 2000

2. Baumann C, Fuchs H, Kiwit J, Westphalen K, Hierholzer J: Complications in percutaneous vertebroplasty associated with puncture or cement leakage. Cardiovasc Intervent Radiol 30: 161-168, 2007

3. Brodano GB, Amendola L, Martikos K, Bettuzzi C, Boriani L, Gasbarrini A, et al.: Vertebroplasty: benefits are more than risks in selected and evidence-based informed patients. A retrospective study of 59 cases. Eur Spine J 20:1265-1271, 2011

4. Buchbinder R, Osborne RH, Ebeling PR, Wark JD, Mitchell $\mathrm{P}$, Wriedt $\mathrm{C}$, et al.: A randomized trial of vertebroplasty for painful osteoporotic vertebral fractures. N Engl J Med 361: 557-568, 2009

5. Clark W, Bird P, Gonski P, Diamond TH, Smerdely P, McNeil $\mathrm{HP}$, et al.: Safety and efficacy of vertebroplasty for acute painful osteoporotic fractures (VAPOUR): a multicentre, randomised, double-blind, placebo-controlled trial. Lancet 388:1408- 
1416, 2016

6. Diamond TH, Champion B, Clark WA: Management of acute osteoporotic vertebral fractures: a nonrandomized trial comparing percutaneous vertebroplasty with conservative therapy. Am J Med 114:257-265, 2003

7. Dong R, Chen L, Tang T, Gu Y, Luo Z, Shi Q, et al.: Pain reduction following vertebroplasty and kyphoplasty. Int Orthop 37:83-87, 2013

8. Evans AJ, Jensen ME, Kip KE, DeNardo AJ, Lawler GJ, Negin GA, et al.: Vertebral compression fractures: pain reduction and improvement in functional mobility after percutaneous polymethylmethacrylate vertebroplasty retrospective report of 245 cases. Radiology 226:366-372, 2003

9. Firanescu CE, de Vries J, Lodder P, Schoemaker MC, Smeets AJ, Donga E, et al.: Percutaneous vertebroplasty is no risk factor for new vertebral fractures and protects against further height loss (VERTOS IV). Cardiovasc Intervent Radiol 42:9911000, 2019

10. Firanescu CE, de Vries J, Lodder P, Venmans A, Schoemaker MC, Smeets AJ, et al.: Vertebroplasty versus sham procedure for painful acute osteoporotic vertebral compression fractures (VERTOS IV): randomised sham controlled clinical trial. BMJ 361:k1551, 2018

11. Galibert P, Deramond H, Rosat P, Le Gars D: Preliminary note on the treatment of vertebral angioma by percutaneous acrylic vertebroplasty. Neurochirurgie 33:166-168, 1987

12. Gangi A, Clark WA: Have recent vertebroplasty trials changed the indications for vertebroplasty? Cardiovasc Intervent Radiol 33:677-680, 2010

13. Johnell O, Kanis JA: An estimate of the worldwide prevalence and disability associated with osteoporotic fractures. Osteoporos Int 17:1726-1733, 2006

14. Kado DM, Browner WS, Palermo L, Nevitt MC, Genant HK, Cummings SR: Vertebral fractures and mortality in older women: a prospective study. Study of Osteoporotic Fractures Research Group. Arch Intern Med 159:1215-1220, 1999

15. Kallmes DF, Comstock BA, Heagerty PJ, Turner JA, Wilson DJ, Diamond TH, et al.: A randomized trial of vertebroplasty for osteoporotic spinal fractures. N Engl J Med 361:569-579, 2009

16. Klazen CA, Verhaar HJ, Lampmann LE, Juttmann JR, Blonk $\mathrm{MC}$, Jansen FH, et al.: VERTOS II: percutaneous vertebroplasty versus conservative therapy in patients with painful osteoporotic vertebral compression fractures; rationale, objectives and design of a multicenter randomized controlled trial.
Trials 8:33, 2007

17. Kobayakawa T, Miyazaki A, Saito M, Suzuki T, Takahashi J, Nakamura Y: Denosumab versus romosozumab for postmenopausal osteoporosis treatment. Sci Rep 11:11801, 2021

18. Lewis GS, Mischler D, Wee H, Reid JS, Varga P: Finite element analysis of fracture fixation. Curr Osteoporos Rep 19: 403-416, 2021

19. Lim J, Choi SW, Youm JY, Kwon HJ, Kim SH, Koh HS: Posttraumatic delayed vertebral collapse: Kummell's disease. J Korean Neurosurg Soc 61:1-9, 2018

20. Melton LJ, 3rd, Thamer M, Ray NF, Chan JK, Chesnut CH, 3rd, Einhorn TA, et al.: Fractures attributable to osteoporosis: report from the National Osteoporosis Foundation. J Bone Miner Res 12:16-23, 1997

21. Pappou IP, Papadopoulos EC, Swanson AN, Cammisa FP, Jr., Girardi FP: Osteoporotic vertebral fractures and collapse with intravertebral vacuum sign (Kümmel's disease). Orthopedics 31:61-66, 2008

22. Parreira PCS, Maher CG, Megale RZ, March L, Ferreira ML: An overview of clinical guidelines for the management of vertebral compression fracture: a systematic review. Spine J 17:1932-1938, 2017

23. Ryan PJ, Blake G, Herd R, Fogelman I: A clinical profile of back pain and disability in patients with spinal osteoporosis. Bone 15:27-30, 1994

24. Silverman SL: The clinical consequences of vertebral compression fracture. Bone 13 Suppl 2:S27-S31, 1992

25. Stallmeyer MJ, Zoarski GH, Obuchowski AM: Optimizing patient selection in percutaneous vertebroplasty. J Vasc Interv Radiol 14:683-696, 2003

26. Suzuki N, Ogikubo O, Hansson T: The course of the acute vertebral body fragility fracture: its effect on pain, disability and quality of life during 12 months. Eur Spine J 17:13801390, 2008

27. Uebelhart B, Ferrari S: Romosozumab : a new treatment for severe osteoporosis. Rev Med Suisse 17:784-787, 2021

28. Whedon GD: Disuse osteoporosis: physiological aspects. Calcif Tissue Int 36 Suppl 1:S146-S150, 1984

29. Whitehouse RW: (i) Patient selection criteria for vertebroplasty or kyphoplasty in painful osteoporotic fracture. Orthop Trauma 25:79-82, 2011

30. Zhang L, Zhai P: A comparison of percutaneous vertebroplasty versus conservative treatment in terms of treatment effect for osteoporotic vertebral compression fractures: A meta-analysis. Surg Innov 27:19-25, 2020 